\title{
O impacto da depressão pós-parto para a interação mãe-bebê ${ }^{1}$
}

\author{
Daniela Delias de Sousa Schwengber \\ Cesar Augusto Piccinini \\ Universidade Federal do Rio Grande do Sul
}

\begin{abstract}
Resumo
O presente artigo examina algumas questões teóricas e estudos empíricos a respeito do impacto da depressão pós-parto para a interação mãe-bebê. Analisam-se as características da depressão pós-parto e fatores de risco associados à sua ocorrência. Discutem-se, em particular, as repercussões do estado depressivo da mãe para a qualidade da interação com o bebê e, conseqüentemente, para o desenvolvimento posterior da criança. Os estudos revisados sugerem que a depressão pós-parto afeta a qualidade da interação mãe-bebê, especialmente no que se refere ao prejuízo na responsividade materna. Por outro lado, apontam que os efeitos da depressão da mãe na interação com o bebê dependem de uma série de fatores, o que não permite a realização de um prognóstico baseado em fatores isolados.
\end{abstract}

Palavras-chave: depressão pós-parto; interação mãe-bebê; desenvolvimento sócio-emocional

\begin{abstract}
The impact of postpartum depression to mother-child interaction. The present article examines some theoretical aspects and empirical studies related to the impact of postpartum depression to mother-child interaction. The characteristics of postpartum depression and the risk factors related to its occurrence are also examined. Furthermore, it discusses the consequences of maternal depressed state to mother-child interaction and to child development. The revised studies suggest that postpartum depression negatively affects the motherchild interaction quality, particularly maternal responsivity. On the other hand, studies show that postpartum depression effects on mother-child interaction depend on various factors, which makes it impossible for us to make predictions based on any isolated factor.
\end{abstract}

Key words: postpartum depression; mother-child interaction; socio-emotional development

A interação mãe-bebê e sua relação com o desenvolvimento posterior da criança representam uma área de grande interesse entre os pesquisadores do desenvolvimento infantil. As investigações conduzidas nesse sentido têm enfatizado tanto as contribuições maternas quanto as contribuições do próprio bebê para a qualidade da interação (Brazelton, 1988; Klaus \& Kennel, 1993; Klaus, Kennel \& Klaus, 2000). Dentre os fatores que contribuem com o processo interativo, o papel exercido pela depressão pós-parto tem sido abordado por inúmeras investigações nas últimas décadas, devido às evidências de que o estado depressivo da mãe pode repercutir negativamente no estabelecimento das primeiras interações com o bebê e, em conseqüência, no desenvolvimento afetivo, social e cognitivo da criança (Cummings \& Davies, 1994; Dodge, 1990; Field, 1998; Tronick \& Weinberg, 1997).
O exame da literatura demonstra que grande parte dos estudos que versam sobre a interação bebê-mãe deprimida aborda as implicações da depressão pós-parto para a interação nos primeiros meses após o nascimento da criança, considerando-se a maior incidência dos quadros depressivos nesse período (Campbell, Cohn \& Meyers, 1995). No entanto, evidências de que muitas mulheres permanecem com os sintomas por um período prolongado enquanto outras começam a se sentir deprimidas mais tardiamente no primeiro ano após o parto apontam para a necessidade da realização de investigações que abordem o problema em períodos mais avançados do desenvolvimento do bebê (Brown, Lumley, Small \& Astbury, 1994).

Nesse sentido, a presente revisão tem como objetivo contribuir para o estudo da interação mãe-bebê, examinando as investigações que ressaltaram o papel da depressão pós- 
parto no desenvolvimento inicial da criança. Inicialmente, serão examinadas algumas características da depressão pós-parto e examinados alguns fatores associados à sua ocorrência. Em um segundo momento, serão feitas considerações a respeito do impacto da depressão pós-parto para a interação mãebebê. Por fim, serão revisadas suas implicações para o desenvolvimento infantil.

\section{Características da depressão pós-parto e fatores associados à sua ocorrência}

O nascimento de um bebê, principalmente em se tratando do primeiro filho, tem sido considerado por diversos autores como um evento propício ao surgimento de problemas emocionais nos pais, como depressões, psicoses pós-parto e manifestações psicossomáticas (Klaus et al., 2000; Maldonado, 1990; Szejer \& Stewart, 1997). A depressão comumente associada ao nascimento de um bebê refere-se a um conjunto de sintomas que iniciam geralmente entre a quarta e a oitava semana após o parto, atingindo de 10 a 15\% das mulheres. Esses sintomas incluem irritabilidade, choro freqüente, sentimentos de desamparo e desesperança, falta de energia e motivação, desinteresse sexual, transtornos alimentares e do sono, a sensação de ser incapaz de lidar com novas situações, bem como queixas psicossomáticas (Klaus et al., 2000).

Os distúrbios do humor que caracterizam o período pósparto incluem também a melancolia da maternidade (baby blues) e as psicoses puerperais (Souza, Burtet, \& Busnello, 1997). O primeiro quadro, que se caracteriza por um distúrbio de labilidade transitória de humor, atinge cerca de 60\% das novas mães entre o terceiro e o quinto dia após o parto, porém geralmente tem remissão espontânea. Já as psicoses puerperais apresentam sintomas acentuados, os quais freqüentemente requerem tratamento intensivo e, por vezes, hospitalização. A incidência desse quadro, de acordo com os autores, é de apenas dois a quatro casos em cada mil partos, ocorrendo entre as duas primeiras semanas após o parto.

Alguns autores sugerem que, por vezes, os sintomas da depressão pós-parto, podem surgir em algum outro momento do primeiro ano de vida do bebê e não necessariamente nas primeiras semanas após o seu nascimento (Beck, 1991; Brown et al., 1994; Klaus et al., 2000; Murray, Cox, Chapman, \& Jones, 1995). Para esses autores, ainda que o quadro evidencie características específicas nas semanas que se seguem ao nascimento da criança, similaridades em relação à incidência, prevalência, características clínicas e fatores associados sugerem pouca distinção entre as depressões que acometem mães de crianças pequenas, independentemente de suas idades (Beeghly, Weinberg, Olson, Kernan, Riley, \& Tronick, 2002; Cooper, Campbell, Day, Kennerley, \& Bond, 1988; Cox, Murray, \& Chapman, 1993).

A vulnerabilidade da mulher ao desenvolvimento ou agravamento da depressão após o nascimento de um filho tem sido ressaltada em diversos estudos (Hopkins, Marcus, \& Campbell, 1984; O’Hara, Neunaber, \& Zekoski, 1984). De acordo com a literatura, há poucas evidências de que a presença da depressão pós-parto esteja associada apenas a mecanismos biológicos, como, por exemplo, a uma diminuição nos níveis hormonais, o que a explicaria como resultado de alterações metabólicas (Carnes, 1983). Nesse sentido, alguns autores têm enfatizado que uma combinação de fatores biológicos, obstétricos, sociais e psicológicos pode significar risco para a depressão pós-parto (Cooper \& Murray, 1995; Reading \& Reynolds, 2001). De fato, uma série de estudos tem evidenciado uma associação entre a ocorrência da depressão pós-parto e o pouco suporte oferecido pelo parceiro ou por outras pessoas com quem a mãe mantém relacionamento (Beck, 2002; Beck, Reynolds, \& Rutowsky, 1992; Brown et al., 1994; Deal \& Holt, 1998; Kumar \& Robson, 1984; Pfost, Stevens, \& Lum, 1990; Romito, Saurel-Cubizolles, \& Lelong, 1999), o não planejamento da gestação, o nascimento prematuro e a morte do bebê (Kumar \& Robson, 1984), a dificuldade em amamentar (Warner, Appleby, Whitton, \& Faragher, 1996), e a dificuldades no parto (Brown et al., 1994). Além disso, alguns estudos mostram uma associação entre a depressão da mãe e eventos de vida estressantes, como: problemas de saúde da criança (Romito et al., 1999); dificuldades relacionadas ao retorno ao trabalho (Hock \& DeMeis, 1990; Murray et al., 1995); e adversidades sócioeconômicas (Seguin, Potvin, St Denis, \& Loiselle, 1999).

Variáveis sócio-demográficas, como idade, nível educacional e estado civil da mãe não têm apresentado uma associação consistente com a ocorrência da depressão pósparto (Romito et al., 1999). Contudo, entre esses fatores o estado civil tem aparecido em alguns estudos como mais associado a esse quadro especialmente entre mães solteiras sem apoio social (Pfost et al., 1990). Em outro estudo que avaliou a contribuição de fatores sócio-demográficos para a ocorrência da depressão pós-parto entre adolescentes, Deal e Holt (1998) encontraram que a depressão das mães estava associada com a pouca idade, com a raça negra, estado civil de solteira, baixo nível educacional e suporte social inadequado. Apoiando esses resultados, Brown e colaboradores (1994), em uma investigação que avaliou a incidência de depressão e a experiência da maternidade oito e nove meses após o parto, encontraram que a depressão materna nesse período estava associada principalmente com a ausência de um parceiro, com complicações obstétricas e com a insatisfação relacionada aos cuidados recebidos na maternidade. No entanto, nesse estudo, a idade da mãe, seu nível educacional e renda familiar não estiveram associados com a ocorrência de depressão.

Além dos fatores destacados acima, alguns estudos revelaram que história prévia de doença psiquiátrica ou problema psicológico prévio da mãe, incluindo a melancolia da maternidade também predisseram a ocorrência posterior de depressão pós-parto (Beck et al., 1992; Cutrona \& Troutman, 1986; Klaus et al., 2000). Endossando esse ponto de vista, Klaus e colaboradores (2000) afirmaram que a ocorrência de história anterior pessoal ou familiar de depressão aumenta a probabilidade da depressão pós-parto, sem desconsiderarem a importância da contribuição de fatores psicossociais atuais. Cramer e Palácio-Espasa (1993), no entanto, defenderam que o encontro mãe-bebê pode induzir 
uma patologia específica, determinada mais pelas vicissitudes da interação do que por uma patologia preexistente da mãe.

Alguns autores ressaltaram o caráter conflituoso da experiência da maternidade como um fator de risco para a ocorrência de distúrbios mentais após o nascimento de um bebê (Maldonado, 1990; Soifer, 1980). Nesse sentido, conceberam que tais distúrbios podem ter origem no conflito da mulher em assumir o papel materno, o que tornaria necessário um redimensionamento da própria identidade. Da mesma forma, Stern (1997) afirmou que, com a chegada do bebê, a nova mãe percebe-se diante de uma reelaboração de esquemas a respeito de si mesma, os quais englobam todos os aspectos do seu ser. A reavaliação de sua identidade, sob essa ótica, pode ser acompanhada de um sentimento de perda subjacente ao sentimento de ganhos com a maternidade, o qual pode estar relacionado à presença de sintomas depressivos.

Embora a experiência da maternidade de mulheres que apresentam sintomas depressivos após o nascimento do bebê seja ainda pouco explorada, alguns estudos que investigaram esse tema foram consistentes ao mostrar que mães deprimidas comumente relatam mais dificuldades em exercer a maternidade do que mães não-deprimidas (Downey \& Coyne, 1990; Hock \& DeMeis, 1990; Lovejoy, Graczyk, O’Hare, \& Neuman, 2000; McKim, Cramer, Stuart, \& O’Connor, 1999; Rutter, 1990). Alguns desses estudos mostraram que mães deprimidas definiram-se como menos competentes, menos ligadas emocionalmente às suas crianças, mais dependentes e isoladas socialmente (Milgron \& McCloud, 1996), relataram menos confiança e satisfação com o desempenho do papel materno do que mães não-deprimidas (Anderson, Fleming, \& Steiner, 1994; Brown et al., 1994; Fowles, 1996; Panzarine, Slater, \& Scharps, 1995), e tenderam a descrever seus bebês como crianças com temperamento difícil (Brown et al., 1994; Campbell \& Marcus, 1987; Mebert, 1991; Whiffen, 1990).

Alguns autores apontaram para a contribuição do temperamento do bebê na precipitação da depressão materna, mostrando que, diante de um bebê mais difícil, as mães perceberam a si mesmas como desempenhando uma maternagem mais pobre (Cutrona \& Troutman, 1986). Endossando essa concepção, Murray, Stanley, Hooper, King e Fiori-Cowley (1996), ao avaliarem o comportamento neonatal de bebês de mães primíparas que na gestação foram avaliadas como tendo risco para o desenvolvimento de depressão após o nascimento do bebê, encontraram que funcionamento motor pobre e alto nível de irritabilidade dos bebês entre o décimo e o décimo quinto dia após o nascimento estiveram associados ao início da depressão da mãe dois meses depois do parto. De acordo com os autores, esses resultados indicam que fatores neonatais podem causar um impacto significativo no estado mental da mãe. Contudo, ao avaliarem a contribuição do temperamento do bebê na precipitação da depressão da mãe, Cummings e Davies (1993) chamaram a atenção para a necessidade da realização de estudos que avaliassem o comportamento neonatal antes da ocorrência da depressão da mãe. Os autores consideraram que os resultados encontrados por Cutrona e Troutman deveriam ser interpretados com cautela, tendo em vista a possibilidade de que os bebês já sofressem o impacto das alterações do estilo interativo da mãe deprimida, uma vez que estavam com dois meses de idade no momento da investigação.

Como pôde ser visto, os estudos revisados indicam que a ocorrência da depressão pós-parto está associada a uma série de fatores biológicos, obstétricos, sociais e psicológicos que se inter-relacionam. Além disso, a literatura aponta também para o caráter conflituoso da experiência da maternidade como um fator de risco para a depressão da mãe, uma vez que a maternidade implicaria na assunção de novos papéis e em mudanças profundas na identidade da mulher. Os estudos sugerem também que mães deprimidas tendem a perceber a própria experiência de forma mais negativa do que mães não-deprimidas. Tendo em vista essas concepções, a seguir será examinado o papel da depressão pós-parto na interação mãe-bebê.

\section{Depressão pós-parto e interação mãe-bebê}

Muitos pesquisadores contribuíram para a investigação do papel da depressão pós-parto na interação mãe-bebê. Por exemplo, Spitz (1979), ao tratar da relação entre depressão pós-parto e coprofagia, assinalou que a “perda” da mãe que entra em depressão não é uma perda física, como quando a mãe morre ou desaparece. Segundo o autor, trata-se de uma perda emocional, na medida em que a mãe, ao mudar sua atitude emocional, altera os signos que a identificavam como um “objeto bom” para a criança. Para o autor, mesmo que a mãe se mantenha fisicamente como era, o objeto afetivamente investido pelo bebê está perdido com a depressão, na medida em que a mãe mostra-se emocionalmente mais distante. Tal perda, segundo Spitz, só pode ser vivenciada sob forma semelhante no primeiro ano de vida, sendo específica desse período.

O caráter natural da depressão experimentada por algumas mães durante o período inicial de recuperação após o parto foi ressaltado por Brazelton (1988). Essa depressão materna, segundo o autor, apresenta um lado positivo: a hipersensibilidade da mãe contribui para que ela procure meios de compreender seu bebê. Como se fosse um consolo para seus sentimentos de desorganização, a mãe busca um comportamento organizado no seu bebê. Brazelton ressaltou ainda que a depressão pode também ser útil no sentido de auxiliar a mãe a se afastar do seu antigo mundo, com o que ganha tempo para desenvolver sensibilidade e responsividade com seu bebê. Contudo, Brazelton e Cramer (1992) salientaram que a depressão materna no período pós-parto também se constitui em um dos mais freqüentes fatores que afetam a contingência da interação mãe-bebê. Os autores mostraram que até mesmo as formas mais brandas de depressão da mãe podem afetar o bebê, na medida em que ele perceberia as mínimas deficiências na contingência no comportamento materno.

Para alguns autores, as depressões maternas contemporâneas se apresentam algumas vezes sob formas pouco evidentes (Mazet \& Stoleru, 1990). Os quadros são mais tórpidos e latentes, camuflados sob a máscara da astenia e 
queixas somáticas, as quais estão relacionadas à pobreza da interação, à ausência ou a pouca harmonização afetiva. Mazet e Stoleru salientaram que as mães deprimidas são inseguras em suas capacidades maternas, o que leva a um afrouxamento da atenção da criança e ao desvio do olhar, característico de uma microrejeição. Já a mãe, sentindo-se rejeitada, suspende a interação, no sentido de uma parada momentânea que tem o objetivo de facilitar o reajustamento do seu comportamento. Com isso, as seqüências interativas terminam rapidamente.

A experiência interativa entre bebês e mães deprimidas foi caracterizada por Stern (1997) em termos de microeventos, descritos como aqueles eventos triviais e de curta duração que causam um impacto momentâneo no bebê. Como exemplo do que seriam microeventos, Stern destacou o que uma mãe faz com os olhos e o rosto no exato momento em que seu bebê lhe dirige um sorriso. Nessa perspectiva, a depressão torna-se familiar ao bebê na forma de muitos microeventos repetidos, ou seja: quando as mães ficam deprimidas não ocorre uma mudança brutal, mas um processo progressivo de desligamento, o qual geralmente é parcial. Para o autor, a experiência de estar com uma mãe deprimida caracteriza-se por pelo menos quatro experiências subjetivas: a primeira se refere à já citada experiência do bebê de microdepressão repetida, o que ocorre quando a mãe sob depressão rompe o contato visual com o bebê e não tenta restabelecê-lo. A partir do fracasso nas suas tentativas de ter a mãe emocionalmente presente, o bebê tenta a proximidade através da identificação e da imitação. A segunda experiência subjetiva a que se refere Stern é caracterizada pela experiência do bebê como um reanimador. Ela foi baseada em evidências de que o bebê, diante de uma situação de microdepressão, tenta fazer com que a mãe volte à vida, o que muitas vezes funciona, já que a depressão materna não pode ser considerada total, nem tampouco constante. A terceira experiência subjetiva do bebê no sentido de estar com a mãe refere-se à visão que o bebê tem da mãe como estando em segundo plano quando da sua busca de outras formas de estimulação, ou seja: se as tentativas de reanimar a mãe falham, o bebê parte em busca de um nível mais apropriado de estimulação e interesse no mundo. Por fim, a quarta experiência subjetiva de estar com uma mãe deprimida refere-se, segundo Stern, ao desejo do bebê de estar com a mãe não-deprimida. Este esquema de estar com a mãe não-deprimida tem como ponto de partida o esforço da mãe deprimida, geralmente manifestado em rompantes, para estar com o filho. O resultado de tal esforço consiste em uma certa falta de autenticidade, que culmina em uma falsa interação entre uma falsa mãe e um falso self. Stern ressaltou que o bebê consegue discriminar as discrepâncias nesse tipo de interação, mas acaba por aceitar o que a mãe oferece, dado o seu desejo de uma interação mais viva com a mãe.

As concepções de Stern (1997) foram baseadas tanto na sua atividade como psicoterapeuta e pesquisador de díades mãe-bebê, como em uma série de estudos que, nos últimos anos, exploraram cada vez mais as interações entre bebês e mães deprimidas, a partir da observação das interações facea-face estabelecidas entre as díades. Os resultados mostraram que mães deprimidas, quando comparadas às mães não- deprimidas, gastam menos tempo olhando, tocando e falando com seus bebês, apresentam mais expressões negativas do que positivas, mostram menos responsividade contingente, menos espontaneidade e menores níveis de atividade (Cohn, Campbell, Matias, \& Hopkins, 1990; Field, 1984; Field et al., 1985; Field et al.; 1988). Por sua vez, bebês de mães deprimidas quando comparados aos de não-deprimidas, exibem menos afeto positivo e mais afeto negativo, menor nível de atividade, menos vocalização, costumam distanciar o olhar, apresentam mais aborrecimento, protestos mais intensos, mais expressões de tristeza e raiva, menos expressões de interesse e uma aparência depressiva com poucos meses de idade (Cohn \& cols., 1990; Field, 1984; Pickens \& Field, 1993).

Discutindo esses achados de observações de interação face-a-face, Field, Healy, Goldstein e Guthertz (1990) assinalaram que, em interações normais, a mãe cuidadosa modula o próprio comportamento no sentido de proporcionar ao bebê estimulação adequada, o que caracteriza uma interação sincrônica. Já a depressão pós-parto contribui para que os comportamentos afetivos e de atenção da díade mãebebê tornem-se assincrônicos, na medida em que a mãe encontra-se afetivamente não-responsiva. Com isso, o bebê tende a experimentar uma desorganização comportamental, diminuindo o nível de respostas contingentes em relação à mãe. Essa diminuição, segundo os autores, reflete o aprendizado do bebê de que seu comportamento exerce um mínimo efeito no comportamento materno. Essa reação do bebê, por sua vez, pode aumentar a frustração materna, fazendo com que a mãe utilize estratégias intrusivas no sentido de obter respostas de seu filho.

Embora os estudos acima apontem para particularidades da interação bebê-mãe deprimida, ainda não está claro o quanto bebês com comportamento depressivo, filhos de mães deprimidas, generalizam esse comportamento com outras pessoas familiares não-deprimidas. Algumas evidências mostram que o comportamento depressivo desses bebês pode também ser observado em interações face-a-face com adultos não-deprimidos. Por exemplo, o estudo desenvolvido por Field e colaboradores (1988) revelou que pessoas que não sabiam que estavam interagindo com bebês de mães deprimidas mostraram afeto negativo e baixo nível de atividade ao interagirem com estes bebês. Esses resultados sugerem que o comportamento depressivo dos bebês pode reforçar um comportamento materno depressivo. Contudo, outros autores encontraram evidências contrárias, indicando que bebês de mães deprimidas interagiam melhor com adultos nãodeprimidos. Hossain, Field, Gonzalez, Malphurs e Delvalle (1994) encontraram uma melhor interação entre bebês e seus pais não-deprimidos do que com a mãe deprimida, ao passo que não havia diferença na interação entre mães nãodeprimidas e pais não-deprimidos. Estudo semelhante realizado por Pelaez-Nogueras, Field, Cigales, Gonzalez e Clasky (1994) mostrou que os comportamentos de bebês depressivos foram diferenciados quando interagiram com mulheres não-deprimidas, sugerindo que os bebês respondiam diferentemente a adultos deprimidos e não-deprimidos que lhes eram familiares. 
As evidências de que as interações face-a-face entre bebês e suas mães diferem em função da depressão pósparto sugerem que o estado afetivo da mãe pode repercutir também nas suas vocalizações para o bebê. Bettes (1988) avaliou as implicações da depressão pós-parto em relação à duas características da linguagem materna: tempo de expressão vocal e pausas e o uso de entonação da voz. A autora verificou que mães deprimidas apresentavam vocalizações significativamente mais baixas para responder às vocalizações de bebês de quatro meses, assim como apresentavam mais variações vocais e pausas, e eram menos aptas a utilizar a entonação exagerada que é característica da linguagem materna.

A literatura a respeito do papel da depressão pós-parto na interação mãe-bebê tem focalizado principalmente os primeiros meses de vida do bebê. Recentemente, os efeitos da intensidade e duração da depressão pós-parto também têm sido investigados. Campbell et al. (1995) demonstraram que o impacto da depressão na interação face-a-face estava associado ao tempo de permanência do diagnóstico. Os autores filmaram a interação entre mães deprimidas e nãodeprimidas com seus bebês aos dois, quatro e seis meses de idade. Os resultados não revelaram diferenças na interação de mães deprimidas e não-deprimidas com seus bebês de dois meses. Contudo, aos seis meses mães deprimidas foram menos positivas com seus bebês, assim como os bebês mostraram-se menos positivos. Endossando esses achados, Field (1995) verificou que mães que estiveram deprimidas durante os primeiros meses após o parto, mas cuja depressão não persistiu além dos seis meses de vida do bebê, tiveram crianças que também não se mostraram deprimidas na interação aos doze meses. Porém, mães que continuaram deprimidas além dos seis primeiros meses tiveram bebês que demonstraram ter desenvolvido um "estilo" depressivo na interação com um ano de idade. Com base nesses resultados, os autores chamaram a atenção para a necessidade de se distinguir entre depressão transitória e prolongada quando se avaliam os efeitos da depressão pós-parto na interação mãe-bebê.

As evidências de que os efeitos da depressão pós-parto para a interação mãe-bebê dependem também da cronicidade do quadro depressivo têm levado a que se avalie o seu impacto em momentos posteriores do desenvolvimento do bebê. Os estudos realizados nessa perspectiva não utilizaram observações da interação face-a-face, mas, sim, situações de jogo nas quais as habilidades e comportamentos mais sofisticados da criança pudessem ser observados.

Um aspecto avaliado nesses estudos refere-se à atenção compartilhada que a díade estabelece no momento da interação. A atenção compartilhada está relacionada ao desenvolvimento do vocabulário, sendo a base para o crescimento da comunicação e, consequentemente, dos relacionamentos (Goodman \& Brumley, 1990). Os estudos desenvolvidos nesse sentido sugeriram que mães deprimidas podem ser menos aptas a coordenar um foco de atenção com seus filhos, na medida em que se mostraram mais preocupadas e pouco atentas às suas crianças (Goldsmith \& Rogoff, 1997;
Goodman \& Brumley, 1990). Por exemplo, em uma investigação que comparou os modelos de atenção entre díades com mães deprimidas e não-deprimidas em uma situação que permitia a utilização tanto de um foco de atenção em comum como um foco de atenção independente, Goldsmith e Rogoff (1997) concluíram que mães deprimidas prestavam menos atenção a um evento em comum com suas crianças de dezoito a trinta meses do que mães não-deprimidas, tanto em termos de seu foco de atenção como em relação às suas atividades com a criança.

As expressões afetivas das díades durante a interação mãe-bebê também foram investigadas em alguns estudos sobre o impacto da depressão da mãe em estágios mais avançados do desenvolvimento do bebê (Hart, Jones, Field, \& Lundy, 1999; Radke-Yarrow, Nottelmann, Belmont, \& Welsh, 1993; Seiner \& Gelfand, 1995). Em um desses estudos, os autores examinaram diferenças na ocorrência de algumas expressões de afeto de mães deprimidas e não-deprimidas e de seus bebês (Radke-Yarrow, Nottelmann, Belmont, \& Welsh, 1993). Os resultados revelaram que mães deprimidas mostraram mais afeto negativo do que mães não-deprimidas e apontaram também para uma correlação entre o afeto das mães e dos bebês. Para os autores, o afeto materno deve ser avaliado como uma parte inseparável das manifestações comportamentais da mãe em relação à criança, mesmo que constitua apenas uma dimensão da depressão que interfere na parentalidade.

Em outro estudo, que examinou o efeito da simulação materna de depressão e afastamento para a interação mãebebê, as díades foram observadas em episódios interativos nos quais as mães simulavam por vezes afeto depressivo e afastamento e, em outro momento, afeto normal (Seiner \& Gelfand, 1995). Os resultados mostraram que, diante das mães menos responsivas, expressivas, envolvidas e falantes, os bebês tendiam a se afastar fisicamente e apresentavam mais comportamentos negativos para chamar atenção. Quando as mães não estavam simulando afeto depressivo e afastamento, os bebês evidenciavam mais comportamentos positivos e brincavam mais próximos às mães.

Mais recentemente, o impacto da depressão materna na exploração de brinquedos pelos bebês no final do primeiro ano de vida tem sido examinado, em virtude das evidências de que essa exploração estaria associada ao desenvolvimento cognitivo posterior da criança (Hart, Field, Delvalle, \& PelaezNogueras, 1998; Lawson, Parrinello, \& Ruff, 1992; TamisLeMonda \& Bornstein, 1989). Mais especificamente, os autores apontaram que comportamentos maternos de introduzir brinquedos e manter a atenção do bebê em um brinquedo pelo qual mostrou interesse estariam associados a um aumento na exploração de objetos por parte do bebê enquanto que o redirecionamento de sua atenção e a manifestação de intrusividade estariam relacionados a um decréscimo nessa exploração.

Em um desses estudos, Hart, Field, Delvalle e PelaezNogueras (1998) investigaram diferenças na interação mãebebê de díades com mães deprimidas e não-deprimidas em relação aos comportamentos associados com a exploração de objetos pelo bebê. Os autores encontraram que os bebês 
de mães deprimidas mostraram-se menos engajados na exploração de objetos e que filhas de mães deprimidas apresentaram mais afeto negativo. Além disso, encontraram que mães deprimidas cujos bebês eram meninos foram mais intrusivas do que mães não-deprimidas. Os resultados revelaram também que, embora não tenham sido encontradas diferenças entre os grupos quanto à introdução de brinquedos, mães deprimidas introduziam mais brinquedos para meninos do que para meninas. Esses resultados corroboraram evidências a respeito da maior intrusividade e envolvimento ativo na interação das mães deprimidas com meninos (Radke-Yarrow et al., 1995).

Em um estudo semelhante realizado por Hart, Jones, Field e Lundy (1999), os autores chamaram a atenção para a existência de dois estilos distintos que uma mãe deprimida pode desenvolver em interação com seu filho: o primeiro é caracterizado pelo afastamento, falta de engajamento e pouca estimulação; o segundo, caracteriza-se por comportamentos intrusivos e de superestimulação. Os resultados mostraram que mães deprimidas intrusivas apresentavam mais respostas positivas, mais demonstração de brinquedos e uma tendência maior a guiarem fisicamente seus bebês que, no entanto, mostraram menos manipulação de objeto. Por outro lado, mães deprimidas que se mostraram mais apáticas, quietas e afastadas mantinham a criança brincando com mais freqüência e mostravam afeto mais restrito enquanto suas crianças demonstraram menos expressão afetiva, positiva ou não. Segundo os autores, os resultados sugerem que a exposição da criança a estilos de interação não adequados, como ocorre com mães deprimidas, representa diferentes tipos de risco para o desenvolvimento cognitivo e afetivo da criança. Uma vez analisados de forma conjunta, estes estudos apontam para diversos efeitos da depressão da mãe na interação com o bebê tanto nos primeiros meses após o parto como em momentos mais avançados do desenvolvimento. Tendo em vista que o estado depressivo da mãe pode repercutir negativamente no desenvolvimento do bebê, a seguir serão revisados os estudos que investigaram a relação entre a depressão pós-parto e o desenvolvimento infantil.

\section{Depressão pós-parto e desenvolvimento infantil}

A literatura aponta que crianças de pais deprimidos têm de duas a cinco vezes maior possibilidade de desenvolver problemas emocionais e de comportamento (Dodge, 1990). O impacto da depressão pós-parto, de acordo com Cummings e Davies (1994), deve ser considerado dentro de um contexto familiar mais amplo, no qual atuam elementos interdependentes. Nesse sentido, o modelo de compreensão das implicações da depressão da mãe para o desenvolvimento infantil proposto pelos autores considerou as características maternas, as relações mãe-criança, o funcionamento do casal e as características da criança. Para os autores, o impacto da depressão na criança vai depender de como esta afeta o comportamento, a cognição e as emoções da própria mãe. Nesta perspectiva, a depressão afeta a criança pela alteração dos modelos de interação mãe-criança ou pelo aumento da discórdia entre o casal, que tem efeitos negativos no desenvolvimento infantil. De qualquer modo, a criança não é considerada um recipiente passivo dos estímulos ambientais, mas, sim, um participante ativo na formação de suas trajetórias de desenvolvimento e nos efeitos dessas trajetórias. Cummings e Davies ressaltaram também a importância da transmissão genética no desenvolvimento de problemas emocionais e comportamentais na criança, concebendo, no entanto, que outros mecanismos operam igualmente bem neste sentido: o ambiente e os efeitos da interação. Nessa perspectiva, a transmissão da psicopatologia dos pais para a criança ocorreria na medida em que a depressão parental leva a uma desorganização na parentalidade e no ambiente familiar, que, por sua vez, conduz ao funcionamento mal-adaptativo da criança.

Ao situar a interação com a mãe como o caminho pelo qual a maioria das influências do mundo chega ao bebê, Stern (1997) também concebeu a psicopatologia infantil em termos de problemas que ocorrem na interação mãe-bebê. Para o autor, influências patogênicas poderiam estar associadas a fatores sociais, econômicos e culturais, mas só teriam um impacto sobre o bebê na medida em que influenciassem a díade mãebebê. Portanto, fatores externos que poderiam afetar a saúde mental posterior da criança teriam significado apenas quando traduzidos na linguagem interativa. Mazet e Stoleru (1990) também caracterizaram as psicopatologias que envolvem recém-nascidos como sendo perturbações interativas, na medida em que os transtornos do bebê não se encontram organizados em uma estrutura psicopatológica clara, mas tendem a ser construídos na relação mãe-bebê. Sendo assim, afirmaram que a etiologia das perturbações pode estar ligada a fatores próprios da criança, à patologia prévia dos pais, ou a uma combinação de ambos. Da mesma forma, ao defenderem uma abordagem interdisciplinar das perturbações apresentadas pelo bebê, Brazelton e Cramer (1992) afirmaram que a psicopatologia do bebê relaciona-se à psicopatologia da interação, devendo ser considerados igualmente na investigação dos sintomas o que se mostra observável e o cenário fantasmático por trás da manifestação da desordem.

Alguns estudos revelaram uma relação entre a ocorrência de depressão pós-parto e relatos sobre problemas de comportamento da criança aos vinte e oito e trinta e seis meses (Leadbeater, Bishop, \& Raver, 1996) e aos quatro anos de idade (Caplan et al., 1989) entre mães que mantiveram os sintomas nesse período. Da mesma forma, estudos têm apontado para o impacto da depressão pós-parto no desenvolvimento cognitivo da criança, sugerindo uma associação entre distúrbios na qualidade da interação com a mãe deprimida no primeiro ano de vida do bebê e baixo desenvolvimento cognitivo aos dezoito meses (Murray, FioriCowley, Hooper, \& Cooper, 1996) e aos quatro anos de idade (Hay \& Kumar, 1995).

O impacto da depressão pós-parto para a competência social da criança foi examinado em uma investigação conduzida por Goodman, Brogan, Lynch e Fielding (1993), a qual evidenciou que crianças de mães deprimidas foram taxadas como menos populares por seus professores, mas não apresentaram escores menores de autoconceito, competência no autocontrole ou nas habilidades em 
relacionamentos com pares quando comparadas a crianças de mães sem indicadores de depressão. Evidências empíricas apontam também para as implicações da depressão pós-parto na qualidade do apego estabelecido entre a mãe e a criança aos dezoito meses (Murray et al., 1996). Os autores encontraram que mães que estiveram deprimidas dois meses após o nascimento foram menos sensíveis e atentas aos bebês aos dezoito meses. Além disso, mostraram-se menos afirmativas e mais negativas em relação ao desenvolvimento dos bebês. Os autores concluíram que a qualidade do apego era influenciada não só pela depressão materna após o nascimento do bebê, mas também pelas experiências da mãe sobre o seu próprio nascimento e a natureza do relacionamento com sua própria mãe.

Os estudos revisados são consistentes ao afirmar que a depressão materna após o nascimento do bebê implica em importantes conseqüências para o desenvolvimento infantil, especialmente no que se refere à ocorrência posterior de problemas emocionais e de comportamento da criança. Da mesma forma, diversos autores têm enfatizado que a depressão da mãe afeta o bebê ao interferir negativamente na interação estabelecida entre a díade.

\section{Considerações finais}

A revisão da literatura mostra que os quadros depressivos maternos no período pós-parto e ao longo do primeiro ano de vida da criança apresentam algumas particularidades, variando quanto à época de seu surgimento, sua incidência e em relação à severidade dos sintomas. Sabe-se que fatores biológicos, obstétricos, psicológicos e sociais podem contribuir para a precipitação deste quadro que, por vezes, tem uma apresentação atípica, na medida em que a tristeza e a angústia da mãe tendem a ser minimizadas diante dos cuidados intensivos dispensados ao bebê.

A sutileza com a qual os sintomas maternos algumas vezes se apresentam, no entanto, não torna menos importantes suas implicações para a díade mãe-bebê. Mesmo as formas mais brandas de depressão materna podem afetar o bebê, uma vez que ele consegue perceber as mínimas deficiências na contingência no comportamento materno. Dessa forma, os estudos revisados indicam que o estado depressivo da mãe pode repercutir negativamente nas primeiras interações com o bebê e, consequentemente, no desenvolvimento da criança. Como mostram essas investigações, as interações que se estabelecem entre bebês e mães deprimidas caracterizam-se por um menor nível de atividade e sincronia do que aquelas estabelecidas na ausência dos sintomas da depressão materna. Mães deprimidas tendem a ser menos responsivas ao interagirem com seus bebês que, por sua vez, tendem a apresentar mais afeto negativo e menos afeto positivo do que bebês de mães não-deprimidas.

Embora haja um consenso acerca das implicações do estado depressivo da mãe para a relação mãe-bebê, alguns estudos sugerem que o tempo de duração da depressão pós- parto deve ser levado em consideração na avaliação de seus efeitos aversivos, na medida em que a cronicidade dos sintomas maternos estaria relacionada a maiores prejuízos na interação mãe-bebê. Em função disso, o tempo de permanência do diagnóstico de depressão materna tem impulsionado a realização de pesquisas sobre a qualidade da interação mãebebê em períodos posteriores do desenvolvimento infantil, como, por exemplo, a partir do final do primeiro ano de vida. Nessa idade, filhos de mães deprimidas tendem a mostrar menos engajamento na exploração de objetos e também menos expressão de afeto positivo. Algumas dessas mães tendem a ser mais intrusivas, enquanto outras se apresentam menos envolvidas ao brincar com seus filhos.

As evidências apontadas pela literatura indicam a importância da avaliação precoce da depressão já durante a gestação. Uma vez diagnosticado o quadro depressivo da gestante, viabiliza-se a realização de intervenções, sendo um dos objetivos principais o de apoiá-la neste momento importante de transição. Da mesma forma, o diagnóstico da depressão da mãe após o nascimento do bebê representa a possibilidade da realização de intervenções multidisciplinares tão logo os sintomas sejam detectados. Nesse sentido, os profissionais que atuam na área da saúde precisam estar atentos para a importância de intervenções que tragam benefícios à relação mãe-bebê. Sabe-se que os primeiros meses após o parto caracterizam um período bastante sensível para a realização de intervenções com esse objetivo, tendo em vista a gama de sentimentos experimentados pela mãe após o nascimento do bebê. A atuação preventiva das equipes multidisciplinares nesse período pode proporcionar à nova mãe o apoio de que necessita para enfrentar os eventuais episódios de depressão. Mais do que isso, o atendimento precoce à mãe deprimida representa a possibilidade da prevenção do estabelecimento de um padrão negativo de interação com o bebê, o qual pode trazer importantes repercussões para o seu desenvolvimento posterior.

Contudo, é importante assinalar que a presença da depressão em um determinado momento após o nascimento do bebê, por si só, não permite a realização de um prognóstico preciso a respeito de suas implicações na qualidade da interação que se estabelecerá entre a díade nos meses subseqüentes. Nesse sentido, faz-se necessária a realização de estudos que utilizem uma abordagem longitudinal a respeito da depressão pós-parto, a qual leve em conta os diversos fatores que podem contribuir para o seu prolongamento ou remissão. Espera-se que a presente revisão da literatura possa estimular novos estudos e, principalmente, o desenvolvimento de estratégias precoces de intervenção que considerem as particularidades dos quadros depressivos observados a partir do nascimento de um bebê.

\section{Referências}

Anderson, V., Fleming, A., \& Steiner (1994). Mood and the transition to motherhood. Journal of Reproductive and Infant Psychology, 12(2), 69-77.

Beck, C. T. (1991). Maternity blues research: A critical review. Issues in Mental Health Nursing, 12, 291-300. 
Beck, C. T. (2002). Postpartum depression: A metasynthesis. Qualitative Health Research, 12, 453-472.

Beck, C. T., Reynolds, M. A., \& Rutowsky, P. (1992). Maternity blues and postpartum depression. Journal of Obstetric, Gynaecologic and Neonatal Nursing, 21(4), 287-293.

Beeghly, M., Weinberg, M. K., Olson, K. L., Kernan, H., Riley, J., \& Tronick, E. Z. (2002). Stability and change in level of maternal depressive symptomatology during the first postpartum year. Journal of Affective Disorders, 71, 169-180.

Bettes, B. A. (1988). Maternal depression and motherese: Temporal and intonational features. Child Development, 59, 1089-1096.

Brazelton, T. B. (1988). O desenvolvimento do apego: Uma família em formação. Porto Alegre: Artes Médicas.

Brazelton, T. B., \& Cramer, B. G. (1992). As primeiras relações. São Paulo: Martins Fontes.

Brown, G. W., Andrews, B., Harris, T., Adler, Z., \& Bridge, L. (1986). Social support, self-esteem and depression. Psychological Medicine, 16, 813831.

Brown, S., Lumley, J., Small, R., \& Astbury, J. (1994). Missing voices: the experience of motherhood. Nova York: Oxford University Press.

Campbell, S. B., Cohn, J. F., \& Meyers, T. (1995). Depression in first-time mothers: mother-infant interaction and depression chronicity. Developmental Psychology, 31, 349-357.

Caplan, H. L., Cogill, S. R., Alexandra, H., Robson, K. M., Katz, R., \& Kumar, R. (1989). Maternal depression and the emotional development of the child. British Journal of Psychiatry, 154, 818-822.

Carnes, J. W. (1983). Psychosocial disturbances during and after pregnancy: helping the patient cope with prenatal stress and postpartum blues. Postgraduate Medicine, 73(1), 135-145.

Cohn, J. F., Campbell, S. B., Matias, R., \& Hopkins, J. (1990). Face-to-face interactions of postpartum depressed and nondepressed mother-infant pairs at 2 months. Developmental Psychology, 26, 15-23.

Cooper, P. J., Campbell, E. A., Day, A., Kennerley, H., \& Bond, A. (1988). Non-psychotic psychiatric disorder after childbirth: a prospective study of prevalence, incidence, course and nature. British Journal of Psychiatry, 152, 799-806.

Cooper, P. J., \& Murray, L. (1995). The course and recurrence of postnatal depression: evidence for the specificity of the diagnostic concept. British Journal of Psychiatry, 166, 191-195.

Cox, J. L., Murray, D., \& Chapman, G. (1993). A controlled study of the onset, duration and prevalence of postnatal depression. British Journal of Psychiatry, 163, 27-31.

Cramer, B., \& Palácio-Espasa, F. (1993). Técnicas psicoterápicas mãe/bebê. Porto Alegre: Artes Médicas.

Cummings, M. E., \& Davies, P. T. (1994). Maternal depression and child development. Journal of Child Psychology and Psychiatry, 35, 73-112.

Cutrona, C., \& Troutman, B. (1986). Social support, infant temperament, and parenting self-efficacy: a mediational model of postpartum depression. Child Development, 57, 1507-1518.

Deal, L. W., \& Holt, V. L. (1998). Young maternal age and depressive symptoms: results from the 1988 National Maternal and Infant Health Survey. American Journal of Public Health, 88(2), 266-269.

Dodge, K. A. (1990). Developmental psychopathology in children of depressed mothers. Developmental Psychology, 26, 3-6.

Downey, G., \& Coyne, J. C. (1990). Children of depressed parents: an integrative review. Psychological Bulletin, 108, 50-76.

Field, T. (1984). Early interactions between infants and their postpartum depressed mothers. Infant Behavior and Development, 7, 527-532.

Field, T. (1995). Infants of depressed mothers. Infant Behavior and Development, $18,1-13$.

Field, T. (1998). Maternal depression effects on infants and early interventions. Preventive Medicine, 27, 200-203.
Field, T., Healy, B., Goldstein, S., \& Guthertz, M. (1990). Behavior-state matching and synchrony in mother-infant interactions of nondepressed versus depressed dyads. Developmental Psychology, 26, 7-14.

Field, T., Healy, B., Goldstein, S., Perry, S., Bendell, D., Schanberg, S., Zimmerman, E. A., \& Kunh, C. (1988). Infants of depressed mothers show "depressed" behavior even with non-depressed adults. Child Development, 59, 1569-1579.

Field, T., Sandberg, D., Garcia, R., Vega-Lahr, N., Goldstein, S., \& Guy, L. (1985). Pregnancy problems, postpartum depression and early motherinfant interactions. Developmental Psychology, 21, 1152-1156.

Fowles, E. (1996). Relationships among prenatal attachment, presence of postnatal depressive symptoms and maternal role attainment. Journal of the Society of Pediatric Nurses, 1(2), 75-82.

Goldsmith, D. F., \& Rogoff, B. (1997). Mother's and toddler's coordinated joint focus of attention: variations with maternal dysphoric symptoms. Developmental Psychology, 33, 113-119.

Goodman, S. H., Brogan, D., Lynch, M. E., \& Fielding, B. (1993). Social and emotional competence in children of depressed mothers. Child Development, 64, 515-531.

Goodman, S. H., \& Brumley, E. (1990). Schizophrenic and depressed mothers: relational deficits in parenting. Developmental Psychology, 26, 31-39.

Hart, S., Field, T., Del Valle, C., \& Pelaez-Nogueras, M. (1998). Depressed mother's interactions with their one-year-old infants. Infant Behavior and Development, 21, 519-525.

Hart, S., Jones, N. A., Field, T., \& Lundy, B. (1999). One-year-old infants of intrusive and withdraw depressed mothers. Child Psychiatry and Human Development, 30(2), 111-120.

Hay, D. F., \& Kumar, R. (1995). Interpreting the effects of mother's postnatal depression on children's intelligence: a critique and re-analysis. Child Psychiatry and Human Development, 25(3), 165-181.

Hock, E., \& DeMeis, D. (1990). Depression in mothers of infants: the role of maternal employment. Developmental Psychology, 26, 285-291.

Hopkins, J., Campbell, S. B \& Marcus, M. (1987). Role of infant-related stressors in postpartum depression. Journal of Abnormal Psychology, 96, 237-241.

Hopkins, J., Marcus, M., \& Campbell, S. B. (1984). Postpartum depression: a critical review. Psychological Bulletin, 95, 498-515.

Hossain, Z., Field, T., Gonzalez, J., Malphurs, J., \& Del Valle, C. (1994). Infants of depressed mothers interact better with their nondepressed fathers. Infant Mental Health Journal, 15(4), 348-357.

Klaus, M. H., Kennel, J. H., \& Klaus, P. (2000). Vínculo: construindo as bases para um apego seguro e para a independência. Porto Alegre: Artes Médicas.

Kumar, R., \& Robson, K. (1984). A prospective study of emotional disorder in pregnancy and the first postnatal year. British Journal of Psychiatry, 144, 35-47.

Lawson, R., Parrinello, R., \& Ruff, H. (1992). Maternal behavior and infant attention. Infant Behavior and Development, 15, 209-229.

Leadbeater, B. J., Bishop, S., \& Raver, C. C. (1996). Quality of mothertoddler interactions, maternal depressive symptoms, and behavior problems in preschoolers of adolescent mothers. Developmental Psychology, 32, 280-288.

Lovejoy, M. C., Graczyk, P. A., O’Hare, E., \& Neuman, G. (2000). Maternal behavior and parenting behavior: a meta-analytic review. Clinical Psychology Review, 20, 561-592.

Maldonado, M. T. (1990). Psicologia da gravidez: parto e puerpério. Petrópolis: Vozes.

Mazet, P., \& Stoleru, S. (1990). Manual de psicopatologia do recém-nascido. Porto Alegre: Artes Médicas.

McKim, M. K., Cramer, K. M., Stuart, B., \& O’Connor, D. L. (1999). Infant care decisions and attachment security: the Canadian transition to child care study. Canadian Journal of Behavioural Science, 31(2), 92-106. 
Mebert, C. J. (1991). Dimensions of subjectivity in parent's ratings of infant temperament. Child Development, 62, 352-361.

Milgron, J., \& McCloud, P. (1996). Parenting stress and postnatal depression. Stress Medicine, 12(3), 177-186.

Murray, D., Cox, J., Chapman, G., \& Jones, P. (1995). Childbirth: life event or start of a long-term difficulty? British Journal of Psychiatry, 166, 595600.

Murray, L., Fiori-Cowley, A., Hooper, R., \& Cooper, P. (1996). The impact of postnatal depression and associated adversity on early mother-infant interactions and later infant outcome. Child Development, 67, 2512-2526.

Murray, L., Stanley, C., Hooper, R., King, F., \& Fiori-Cowley, A. (1996). The role of infant factors in postnatal depression and mother-infant interactions. Developmental Medicine and Child Neurology, 38, 109-119.

O’Hara, M. W., Neunaber, D. J., \& Zekoski, E. M. (1984). Prospective study of postpartum depression: prevalence, course, and predictive factors. Journal of Abnormal Psychology, 93, 158-171.

Panzarine, S., Slater, E., \& Sharps, P. (1995). Coping, social support and depressive symptoms in adolescent mothers. Journal of Adolescent Health, 17(2), 113-119.

Pelaez-Nogueras, M., Field, T., Cigales, M., Gonzalez, A., \& Clasky, S. (1994). Infants of depressed mothers show less depressed behavior with their nursery teachers. Infant Mental Health Journal, 15(4), 358-367.

Pfost, K. S., Stevens, M. J., \& Lum, C. U. (1990). The relationship of demographic variables, antepartum depression and stress to postpartum depression. Journal of Clinical Psychology, 46, 588-592.

Pickens, J., \& Field, T. (1993). Facial expressivity in infants of depressed mothers. Developmental Psychology, 29, 986-988.

Radke-Yarrow, M., Nottelmann, E., Belmont, B., \& Welsh, J. D. (1993). Affective interactions of depressed and nondepressed mothers and their children. Journal of Abnormal Child Psychology, 21, 683-695.

Reading, R., \& Reynolds, S. (2001). Debt, social disadvantage and maternal depression. Social Science \& Medicine, 53, 441-453.

Romito, P., Saurel-Cubizolles, M. J., \& Lelong, N. (1999). What makes new mothers unhappy: psychological distress one year after birth in Italy and France. Social Science \& Medicine, 49, 1651-1661.
Rutter, M. (1990). Commentary: some focus and process considerations regarding the effects of parental depression on children. Developmental Psychology, 26, 60-67.

Seguin, L., Potvin, L., St-Denis, M., \& Loiselle, J. (1999). Depressive symptoms in the late postpartum among low socioeconomic status women. Birth, 26(3), 157-163.

Seiner, S. H., \& Gelfand, D. M. (1995). Effects of mother's simulated withdrawal and depressed affect on mother-toddler interactions. Child Development, 66, 1519-1528.

Soifer, R. (1980). Psicologia da gravidez: parto e puerpério. Petrópolis: Vozes.

Souza, C. A. C., Burtet, C. M., \& Busnello, E. A. D. (1997). A gravidez como condição de saúde mental e de doença psiquiátrica. Revista Científica Maternidade, Infância e Ginecologia, 17(1), 38-47.

Spitz, R. A. (1979). O primeiro ano de vida. São Paulo: Martins Fontes.

Stern, D. N. (1997). A constelação da maternidade: o panorama da psicoterapia pais/bebê. Porto Alegre: Artes Médicas.

Szejer, M., \& Stewart, R. (1997). Nove meses na vida da mulher: uma abordagem psicanalítica da gravidez e do nascimento. São Paulo: Casa do Psicólogo.

Tamis-LeMonda, C., \& Bornstein, M. (1989). Habituation and maternal encouragement of attention in infancy as predictors of toddler language, play and representational competence. Child Development, 60, 738751.

Tronick, E. Z., \& Weinberg, M. K. (1997). Depressed mothers and infants: failure to form dyadic states of consciousness. In L. Murray \& P. J. Cooper (Orgs.), Postpartum depression and child development (pp. 5481). Nova York: Guilford.

Warner, R., Appleby, L., Whitton, A., \& Faragher, B. (1996). Demographic and obstetric risk factors for postnatal psychiatric morbidity. British Journal of Psychiatry, 168, 607-611.

Whiffen, V. (1990). Maternal depressed mood and perceptions of child temperament. The Journal of Genetic Psychology, 151, 329-339.

1. Este artigo é baseado em parte da dissertação de mestrado de Daniela Delias de Sousa Schwengber, realizada e apresentada no Curso de Pós-Graduação em Psicologia do Desenvolvimento da Universidade Federal do Rio Grande do Sul, sob a supervisão de Cesar Augusto Piccinini.

Daniela Delias de Sousa Schwengber é psicóloga da Secretaria Municipal de Saúde e Bem-Estar de Pelotas, RS, especialista em Psicoterapia Psicanalítica (UCPEL), mestre e doutoranda em Psicologia do Desenvolvimento do Curso de Pós-Graduação em Psicologia do Desenvolvimento da Universidade Federal do Rio Grande do Sul.

Cesar Augusto Piccinini é doutor pela University College of London (Reino Unido), pesquisador do CNPq e professor no Curso de Pós-Graduação em Psicologia do Desenvolvimento da Universidade Federal do Rio Grande do Sul.

Endereço para correspondência: Instituto de Psicologia - UFRGS; Rua Ramiro Barcelos, 2600/111; Porto Alegre, RS; CEP 90035-003. E-mail: daniela.s@pop.com.br 